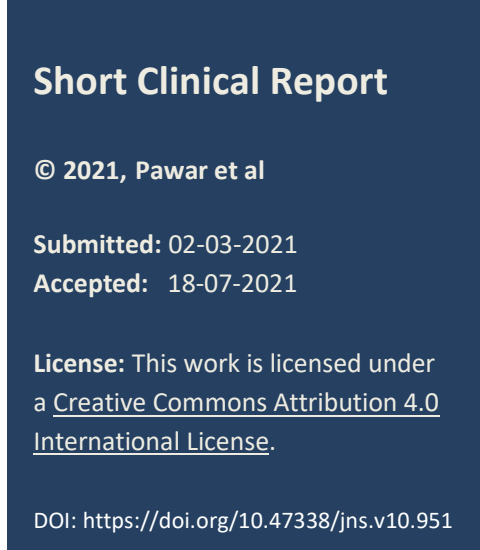

\section{Jejunal atresia with ileojejunal knotting: An unusual presentation}

Naresh Pawar," Pramila Sharma, Priyanka Mittal, Vinita Chaturvedi

Department of Pediatric Surgery, SMS Medical College, Jaipur, Rajasthan, India.

Correspondence*: Naresh Pawar, Mch Resident, Department of Pediatric Surgery, J.K. Lon Hospital, SMS Medical College, Jaipur, Rajasthan, India - 302004. E-mail: nareshp221@gmail.com

\section{CASE PRESENTATION}

A preterm, low birth weight (2450gram) baby born to a primigravida mother was brought to the emergency department with bilious vomiting, abdominal distension, and refusal to feed on day 3 of life. There was a history of the passage of meconium and urine on the same day of birth. On clinical examination, there was abdominal fullness (Fig.1A). The antenatal scan done during the third trimester showed polyhydramnios. There was no history of maternal smoking or medications except for iron and folic acid supplementation. X-ray abdomen erect revealed a gastric shadow with 4-5 air-fluid levels in the center of the upper abdomen with the rest of the abdomen gasless (Fig.1B). The patient was resuscitated with intravenous fluids and broad-spectrum antibiotics. The complete blood count, liver and renal function tests were well within the normal range. On radiological and ultrasonography evaluation there were no components of VACTERL. The baby was kept nil per orally, along with nasogastric tube was placed and planned for emergency surgery.

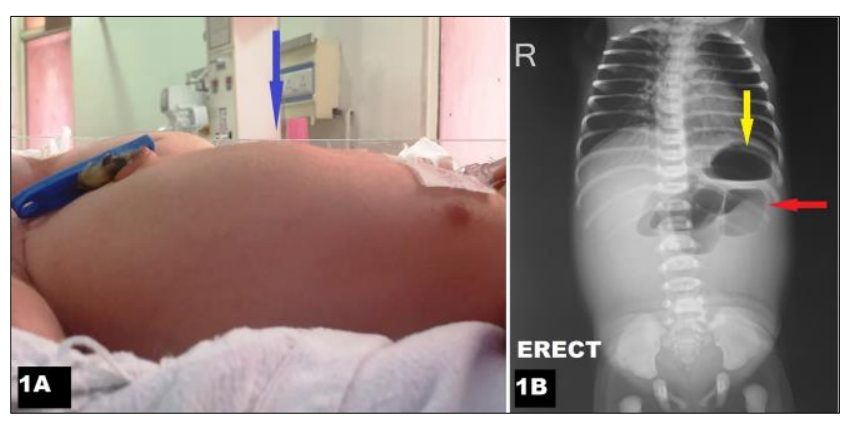

Figure 1: Features at the time of admission. A- Upper abdominal fullness (blue arrow). B. X-ray abdomen erectdilated stomach (yellow arrow) and 4-5 air-fluid levels at the center of the upper abdomen (red arrow) with rest of abdomen gasless.

On exploratory laparotomy, there was type-I jejunal atresia (red arrow) and knotting of proximal dilated jejunum with distal unused ileum (yellow arrow) at
$30 \mathrm{~cm}$ and $15 \mathrm{~cm}$ from duodenojejunal junction respectively (Fig.2A). There was another atresia over proximal dilated jejunum at the site of knotting and formation of blind loop segment of jejunum between two atretic areas (Fig. 2B,2C). The knotting was released, and the blind loop segment of the jejunum was resected with atretic areas $(15 \mathrm{~cm})$. Distal unused bowel patency was checked. Moreover, there was a wide base Meckel's diverticulum which was left as such. (Fig.2D).

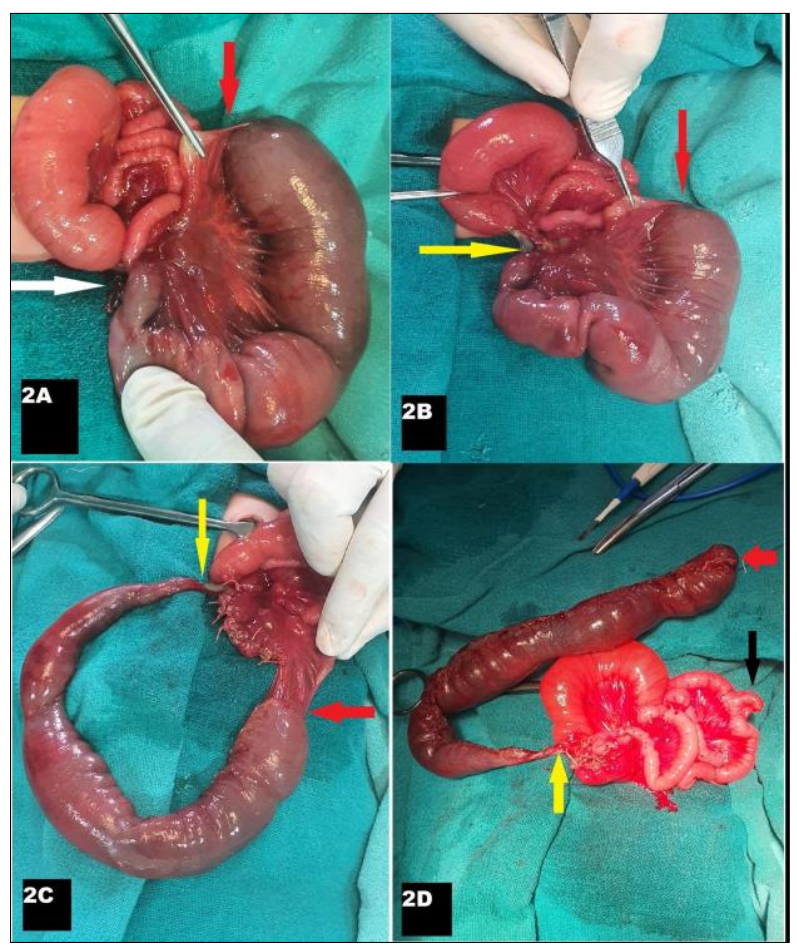

Figure 2: Intraoperative findings. A: type 1 Jejunal atresia (red arrow) and knotting of proximal dilated jejunum by distal unused ileum (white arrow). B \&C: Second atresia over proximal dilated jejunum at the site of knotting (yellow arrow). D: Meckel's diverticulum (black arrow).

A proximal end to distal cut back jejunoileal anastomosis was done in a single layer with polyglycolic acid (PGA) 5-0 round body interrupted 
sutures. The postoperative course remained uneventful. The patient was discharged on day 12 after the removal of skin sutures. On three months follow up; the patient is gaining weight appropriate for age $(5 \mathrm{~kg} / 97 \mathrm{th}$ percentile) on exclusive breastfeeding.

\section{DISCUSSION}

Jejunoileal atresia is a common cause of intestinal obstruction in neonates.[2] The proposed hypothesis of the etiology of jejunoileal atresia is the late intrauterine mesenteric vascular accidents causing ischemic insult to the small bowel.[1] In the literature, the reported causes of ischemic insults leading to small bowel atresia are intrauterine intussusception, perforation, volvulus, internal hernias, tight anterior abdominal wall defects, or thromboembolism.[1-5] The index case had type-I mucosal jejunal atresia in addition to another

\section{REFERENCES}

1. Kumaran N, Shankar KR, Lloyd DA, Losty PD. Trends in the management and outcome of jejuno-ileal atresia. Eur J Pediatr Surg. 2002; 12:163-7. Available from: https://doi.org/10.1055/s-2002-32726.

2. Frischer JS, Azizkhan RG. Jejunoileal Atresia and Stenosis. In: Coron AG, editor. Pediatric Surgery, 7th ed. Philadelphia: Elsevier; 2012. p. 1059-71.

3. Mangray $\mathrm{H}$, Ghimenton F, Aldous C. Jejuno-ileal atresia: its characteristics and peculiarities concerning apple peel atresia, focused on its treatment and outcomes as experienced in one of the leading South African academic centres. Pediatr Surg Int. 2020; proximal mucosal jejunal atresia secondary to the knotting by distal ileal segment. This occurrence has not been reported in English literature so far to the best of our knowledge. With this background of observations, we believe that small bowel knotting can lead to intestinal atresias; and we propose to add this to the existing list of vascular insults leading to atresias.

Acknowledgements: $\mathrm{Nil}$

Conflict of Interest: None declared

Source of Support: Nil

Consent to Publication: Author(s) declared taking informed written consent for the publication of clinical photographs/material (if any used), from the legal guardian of the patient with an understanding that every effort will be made to conceal the identity of the patient, however it cannot be guaranteed.

Author Contributions: Author(s) declared to fulfil authorship criteria as devised by ICMJE and approved the final version.

36:201-7. Available from: https://doi.org/10.1007/ s00383-019-04594-y.

4. Atamanalp SS, Oren D, Yildirgan MI, Basoglu M, Aydinli B, Ozturk G, et al. Ileosigmoidal knotting in children: a review of 9 cases. World J Surg. 2007; 31:31-5. Available from: https://doi.org/10.1007/ s00268-006-0255-6.

5. Beg MY, Bains L, Lal P, Maranna H, Kumar NP. Small bowel knots. Ann R Coll Surg Engl. 2020; 102:571-6. Available from: https://doi.org/10.1308/rcsann. 2020.0122 